\title{
Anisocoria under general anesthesia with Propofol
}

\section{Mahmood Akhlaghi ${ }^{1 *}$}

1. Department of Anesthesiology, Faculty of Medicine, Shahrekord University of Medical Sciences, Shahrekord, Iran

*Corresponding author:Tel: +98 9131811483 Fax: +98 3813334911

Address: Department of Anesthesiology, Shahrekord University of Medical Sciences, Shahrekord, Iran E-mail: akhlaghi236@yahoo.com

Received: 2015/04/21 revised: 2015/05/15 accepted: 2015/06/8

\begin{abstract}
Anisocoria during anesthesia is very rare, but may indicate life-threatening neurologic injuries and create tremendous condition. A 23 -year-old male patient was anesthetized with fentanyl and propofol. Thirty minutes after inducing anesthesia, unilateral mydriasis was detected. The duration of surgery was shortened for prompt evaluation of the pupils. At the end of the operation neither vision problem nor obvious anisocoria was detected in the recovery room. Ophthalmological and neurological consultations revealed no pathological finding and the patient was discharged. Evaluation of the size of the patient's pupils before operation would mitigate the fearful condition resulted from anisocoria.
\end{abstract}

Keywords: Anisocoria, General anesthesia, Propofol

\section{Introduction}

Anisocoria is the disturbance and imbalance of sympathetic and parasympathetic neural pathway which may be life threatening $(1,2)$. Although this finding has been observed following application of some local anesthetic agents or adrenergic drugs, anisocoria per se, results from systemic causes such as neurological and vascular disorders as well as hypoxic injuries to the autonomous system $(1,3)$. During general anesthesia, it is an unusual sign and may indicate neurological problem and could be terrible for anesthesiologists (2). In this tremendous situation, assessing an intraoperative anisocoria according to its cause and evaluating the clinical event and rapid identification could be redemptible for the anesthesiologist. For publication of this report, verbal consent was obtained from the patient.

\section{Case description}

The patient was a 23-year-old male with ASA class II weighing $63 \mathrm{~kg}$, scheduled for open reduction and internal fixation of his left forearm. He was fully alert and had no history of head injury and loss of consciousness according to his chart. On questioning, he had no history of any previous diseases or medication. Preoperatively, the patient's physical examination revealed no abnormal finding except his left forearm fracture, remembering that we did not exam his cornea before induction of anesthesia. Without preoperative medication, the anesthesia plan was a general anesthesia which was induced using $3 \mathrm{mg}$ midazolam, $150 \mu \mathrm{g}$ fentanyl, $150 \mathrm{mg}$ propofol and 30 $\mathrm{mg}$ atracurium. Then the patient was intubated with a cuffed endotracheal tube and underwent mechanical ventilation, and the eyes were kept closed during anesthesia. Anesthesia was maintained by infusing 140 $\mu \mathrm{g} / \mathrm{kg} / \mathrm{min}$ propofol and $0.25 \mu \mathrm{g} / \mathrm{kg} / \mathrm{min}$ alfentanil, and noninvasive blood pressure monitoring, electrocardiogram, pulse oximetry and capnometry were included as monitoring devices. Thirty minutes after inducing anesthesia, as was the 
anesthesiologist's custom, he checked the patient's eyes out and unilateral mydriasis was detected. Examination of the left pupil revealed a markedly dilated pupil $(8 \mathrm{~mm})$ which was non-reactive to direct and consensual light reflex and the right pupil was $3 \mathrm{~mm}$ in diameter and normally reactive to light (Figure 1).

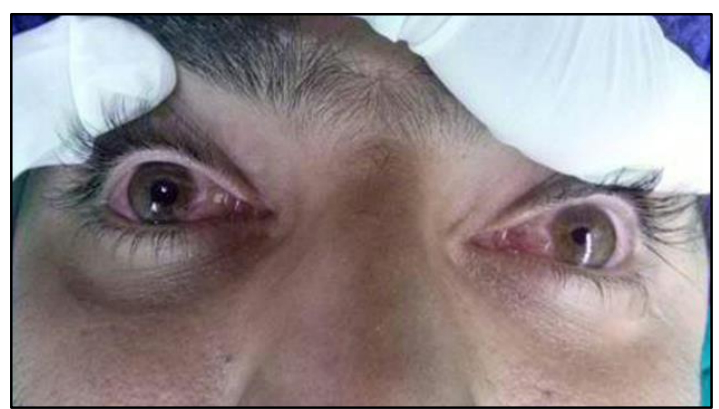

Figure1. Anisocoria under general anesthesia.

As we did not aware of preoperative pupils' situations, we shortened the duration of surgery towards prompt evaluation of the pupils. At the end of the operation the patient was extubated and transferred to the post anesthesia care unit, 45 minutes after inducing anesthesia, being fully conscious. Neither vision problem nor obvious anisocoria was detected in the recovery room and thereafter. Ophthalmological and neurological consultations revealed no pathological finding and the patient was discharged and is doing well.

\section{Discussion}

Anisocoria as an important finding during general anesthesia with propofol is unusual and hardly to be seen. One old study mentioned the incidence of anisocoria as high as 59 percent of patients receiving

\section{References}

1. Nesioonpour S, Khiabani K, Hassanijirdehi M. Unilateral mydriasis after mandibular fracture fixation surgery. Anesth Pain Med. 2014; 4(2):e13831.

2. Inchingolo $\mathrm{F}$, Tatullo $\mathrm{M}$, Abenavoli $\mathrm{FM}$, Marrelli $\mathrm{M}$, Inchingolo $\mathrm{AD}$, Villabruna B, et al. Severe anisocoria after oral surgery under general diethyl ether due to either unequal sensitivity of the pupils to anesthetic agents or to variation in circulation to the pupils or their autonomic nerves (4). We expect to reveal no differences between pupils during induction and maintenance with new intravenous drugs such as propofol. In this case report, anisocoria was revealed during maintaining general anesthesia with propofol and alfentanil which was completely resolved after the end of anesthesia and during the recovery period. For diagnosis of unilateral pupil dilation, the main lethal causes that a clinician should think of are neurologic causes such as cerebrovascular accident, a mass, a cerebral lesion or an ocular trauma. Additionally, accidental exposure to the medications such as atropine and other midriatic agents and local anesthetics, should be considered as pharmacologic causes, which may misdirect and horrify the involved anesthetist. However, our patient had neither systemic nor local causes of anisocoria, and ophtalmological and neurological consultations confirmed the benign process, postoperatively .

Although the finding of perioperative anisocoria may be benign $(1,2,5)$, as in our case whose tremendous situation was terminated postoperatively, it can also indicate life-threatening neurological injury which needs to be intervened, promptly (2, $6,7)$. Preoperative awareness of the pupils' size would mitigate this intraoperative fearful situation. For this reason, we think that, routine preoperative pupillary examination should be considered by anesthesiologists.

anesthesia. Int J Med Sci. 2010; 7(5):314-8.

3. Patel R, Davis C, Sivaswamy L. Anisocoria-not always cause for alarm. J Pediatr. 2014; 164(6):1497.

4. Sobel AM. Anisocoria during general anesthesia. Anesth Analg. 1965; 44(5):522-6. 
5. Yalcin S, Pampal K, Erden A, Oba S, Bilgin S. Do we really need to panic in all anisocoria cases in critical care? Indian J Anaesth. 2010; 54(4):365-6.

6. Aceto P, Perilli V, Vitale E, Sollazzi L. Effect of anesthesia in a patient with pre-existing anisocoria. Eur Rev Med Pharmacol Sci. 2011; 15(2):211-3.

7. Turnbull A, Marsh C. Perioperative anisocoria-the importance of pupil assessment before general anaesthesia. Anaesthesia. 2012; 67(9):1053-4. 